\title{
Ground and excited states of spherically symmetric potentials through an imaginary-time evolution method: Application to spiked harmonic oscillators
}

\author{
Amlan K. Roy* \\ Division of Chemical Sciences, Indian Institute of Science Education and Research Kolkata, \\ Mohanpur Campus, Nadia, 741252, WB, India.
}

\begin{abstract}
Starting from a time-dependent Schrödinger equation, stationary states of 3D central potentials are obtained. An imaginary-time evolution technique coupled with the minimization of energy expectation value, subject to the orthogonality constraint leads to ground and excited states. The desired diffusion equation is solved by means of a finite-difference approach to produce accurate wave functions, energies, probability densities and other expectation values. Applications in case of 3D isotropic harmonic oscillator, Morse as well the spiked harmonic oscillator are made. Comparison with literature data reveals that this is able to produce high-quality and competitive results. The method could be useful for this and other similar potentials of interest in quantum mechanics. Future and outlook of the method is briefly discussed.
\end{abstract}

\footnotetext{
*Email: akroy@iiserkol.ac.in, akroy6k@gmail.com
} 


\section{INTRODUCTION}

Applications of quantum mechanics in various branches of physics, chemistry, biology, such as atomic, molecular, nuclear physics, particle physics, astrophysics, etc., often require solution of Schrödinger equation (SE). The system is characterized by an external potential term present in the Hamiltonian operator. Leaving aside a few occasions, such as the well-known harmonic oscillator or Coulomb potential representing some idealized situations, exact analytical solution in most of these problems remains elusive. Search for such solutions is appealing and have been pursued by a large number of researchers. In recent years, such analytical solutions have been reported for few more potentials such as Kratzer-Fues potential in $\mathrm{N}$ dimension [1], Mie [2], pseudoharmonic potential in 2D [3], 3D [4] and Ndimension [5], Morse [6], Pöschl-Teller [7], Manning-Rosen [8, 9] and some other diatomic molecular potentials [10]. Nevertheless they are few and far between, and it is imperative that alternate approximation methods be developed. Therefore, a variety of accurate, efficient, elegant methodologies for such solutions have been put forth over the years. This encompasses a wide range of analytic, semi-analytic and numerical techniques. The literature is vast; here we refer to some of the most prominent ones, viz., Nikiforov-Uvarov method [11], super-symmetric quantum mechanics [12] asymptotic iteration method [13, 14], exact quantization rule [15, 16], factorization method [17], wave function ansatz approach [18, 19], generalized pseudospectral method [20, 21], proper quantization rule [22, 23], etc.

In all the above mentioned approaches, approximate solutions are obtained starting from a time-independent SE (TISE). In this work, we explore the possibility of an approximate solution based on time-dependent Schrödinger equation (TDSE) instead. This is achieved by transforming the TDSE in imaginary time to a diffusion equation, followed by a minimization of the energy expectation value to reach the global minimum. Such a technique was adopted in connection with a random-walk simulation of the solution of $a b$ initio SE for electronic systems such as, $\mathrm{H}^{2} P, \mathrm{H}_{3}^{+}\left(\mathrm{D}_{3 h}\right){ }^{1} A_{1}, \mathrm{H}_{2}{ }^{3} \Sigma_{u}^{+}, \mathrm{H}_{4}{ }^{1} \Sigma_{g}^{+}$, Be ${ }^{1} S, \mathrm{CH}_{4}$, etc., [24 26]. In a separate work, eigenvalues, eigenfunctions of TDSE were obtained by evolving the same in imaginary time and representing the Hamiltonian in a grid by a relaxation method [27]. Representative applications were given for Morse potential, Hénon-Heiles system and weakly bound states of He on a Pt surface. Another interesting route (the so-called spectral method), based on the grid, to exploit TDSE for obtaining eigenvalues, eigenfunc- 
tions was adopted in [28], whereby the initial wave function was propagated for long time. Then eigenvalues are obtained by performing a Fourier transform of the auto-correlation function of propagated wave with the initial wave function. In yet another development, imaginary-time evolution technique was applied for direct calculation of ground-state densities and other properties of noble gas atoms, ions such as $\mathrm{He}, \mathrm{Be}^{++}, \mathrm{Ne}, \mathrm{Ar}, \mathrm{Kr}, \mathrm{Xe}$, as well as molecules like $\mathrm{H}_{2}, \mathrm{HeH}^{+}, \mathrm{He}_{2}^{++}$, from the solution of a single TD quantum fluid dynamical equation of motion [29 31]. Later, ground as well as excited-state energies, densities and other expectation values of 1D anharmonic and double-well [32], multiple-well [33] and self-interacting nonlinear [34] oscillators were obtained with impressive accuracy through this route. Extension was made to ground and low-lying excited states of double well potentials in 2D [35]. Furthermore, during the same time period, a finite-difference time domain approach was suggested for solution of the respective TDSE in imaginary time. Applications were made to the problems of infinite square potential, quantum anharmonic oscillators in 1D, 2D, 3D, as well as hydrogen atom [36] with reasonable success. Later, this scheme was employed for a charged particle in magnetic field [37], and for the computation of thermal density matrix of a single-particle confined quantum system [38]. An optimized parallelization scheme for solving 3D SE has been presented lately [39]. Imaginary-time propagation technique has also been exploited in numerical solution of eigenvalues, eigenfunctions of large matrices originating from discretization of linear and non-linear SE by means of split-operator method [40], and also for large-scale 2D eigenvalue problems in presence of a magnetic field [41]. Improved, high-order, imaginary-time propagators for 3D SE has been proposed [42, 43], as well as a fourth order algorithm for solving local SE in a homogeneous magnetic field [44].

The purpose of this communication is to present and explore the feasibility of the imaginary time evolution method, as implemented in [29 35], in the context of spherically symmetric 3D potentials. As mentioned above, while for the atoms and molecules in first three references, the diffusion equation originated from an amalgamation of quantum fluid dynamics and density functional theory, which eventually lead to a TD generalized non-linear SE, in latter four references the same arose from the TDSE instead. The current work concern the latter. It is worthwhile mentioning that while ground and excited states were treated in 1D and 2D using this approach [32 35], for spherically symmetric potentials (such as for atoms, as in [29 31], only ground states were attempted. Here we include excited states in 
our study, that can extend its domain of applicability to some other systems. After making some experiments on pedagogical cases like 3D isotropic quantum harmonic oscillator and Morse potential, we focus on the specific case of spiked harmonic oscillator, for illustration. Because of their many interesting properties and considerable challenges they pose, these have been investigated by a number of workers in the past three decades varying in their complexity and accuracy. In particular, we focus on the eigenvalues, position expectation values, radial densities of ground and low-lying excited states. At present, we consider the non-rotational $(\ell=0)$ case, while rotational $(\ell \neq 0)$ situations may be studied in future works. The article is organized as follows. In Section II, we give an outline of the theoretical framework and details of numerical implementation. Obtained results are discussed in Section III along with a comparison with literature results. Finally a few concluding remarks are made in Section V.

\section{THE METHODOLOGY AND NUMERICAL IMPLEMENTATION}

The TDSE of a single particle moving in a time-independent potential field $V(\mathbf{r})$ is:

$$
i \frac{\partial}{\partial t} \psi(\mathbf{r}, t)=H \psi(\mathbf{r}, t)=\left[-\frac{1}{2} \nabla^{2}+V(\mathbf{r})\right] \psi(\mathbf{r}, t)
$$

where $\mathrm{H}$ is the Hamiltonian operator consisting of kinetic and potential energy components. Here and what follows, we employ atomic units. The general solution can be expanded in terms of a set of eigenfunctions $\left\{\phi_{k}\right\}$ and expansion coefficients $\left\{C_{k}\right\}$ as follows:

$$
\psi(\mathbf{r}, t)=\sum_{k=0}^{\infty} C_{k} \phi_{k}(\mathbf{r}) \exp \left(-i \epsilon_{k} t\right) .
$$

The eigenfunctions $\phi_{k}(\mathbf{r})$ and eigenvalues $\epsilon_{k}$ are obtained from the associated TISE. Following previous works (see, for example, [29 35], and references therein), we assume the validity of TDSE in imaginary time $\tau$, and write Eq. (1) in $\tau$. Further, replacing $\tau$ by $-i t$, where $t$ is real time, yields (for brevity, $\psi(\mathbf{r}, t)$ now refers to the diffusion function),

$$
-\frac{\partial \psi(\mathbf{r}, t)}{\partial t}=H \psi(\mathbf{r}, t)
$$

Defining the time differential operator, $D_{t}=\frac{\partial}{\partial t}$, transforms this into a nonlinear diffusionlike equation, which resembles a diffusion-quantum Monte Carlo equation [45],

$$
-D_{t} \psi(\mathbf{r}, t)=H \psi(\mathbf{r}, t)
$$


One may express $\psi(\mathbf{r}, t)$ as,

$$
\psi(\mathbf{r}, t)=C_{0} \phi_{0}(\mathbf{r})+\sum_{k=1}^{\infty} C_{k} \phi_{k}(\mathbf{r}) e^{-\left(\epsilon_{k}-\epsilon_{0}\right) t},
$$

where $\phi_{0}, \epsilon_{0}$ refer to ground-state eigenfunction and eigenvalue. Hence, taking due account of normalization, one observes that, at $t \rightarrow \infty, \psi(\mathbf{r}, t) \rightarrow \phi_{0}$, i.e.,

$$
\lim _{t \rightarrow \infty} \psi(\mathbf{r}, t) \approx C_{0} \phi_{0}(\mathbf{r})
$$

This implies that at any finite non-zero time, $\psi(\mathbf{r}, t)$ can be seen as a linear combination of TISE eigenfunctions $\left\{\phi_{k}\right\}$ with appropriate TD coefficients $\left\{C_{k}\right\}$ which decay exponentially in real time. Therefore, numerically propagating $\psi(\mathbf{r}, t)$ to a sufficiently long time leads to the stationary ground-state wave function (provided $C_{0} \neq 0$ ), apart from a normalization constant, corresponding to the global minimum value of $\langle\psi(\mathbf{r}, t)|H| \psi(\mathbf{r}, t)\rangle$. This is a general technique for solving stationary-state eigenvalue problems in quantum mechanics.

Now let us consider the numerical solution of Eq. (4). In order to accomplish the time propagation of $\psi(\mathbf{r}, t)$, one can use a Taylor expansion of $\psi(\mathbf{r}, t+\Delta t)$ around time $t$,

$$
\psi(\mathbf{r}, t+\Delta t)=\left[1+\Delta t D_{t}+\frac{(\Delta t)^{2}}{2 !} D_{t}^{2}+\cdots\right] \psi(\mathbf{r}, t)=e^{\Delta t D_{t}} \psi(\mathbf{r}, t) .
$$

From Eq. (4), we see that $H=-D_{t}$. Hence the above equation can be rewritten as,

$$
\psi(\mathbf{r}, t+\Delta t)=e^{-\Delta t H} \psi(\mathbf{r}, t)
$$

The time-propagator $e^{-\Delta t H}$ is an evolution operator advancing the diffusion function $\psi(\mathbf{r}, t)$ from an initial time $t$ to next time level $\psi(\mathbf{r}, t+\Delta t)$. It is worth mentioning that, this is a real, non-unitary operator; hence normalization of $\psi(\mathbf{r}, t)$ at an arbitrary time $t$ does not automatically guarantee normalization of $\psi(\mathbf{r}, t+\Delta t)$ at a future time, $t+\Delta t$.

At this point, we focus on the important case of central force, which is derived from a potential energy function that is spherically symmetric, i.e., $V(\mathbf{r})=V(r)$. For this, we discretize the radial variable $r$ (of spherical polar coordinates) according to the following,

$$
\begin{aligned}
& r_{j}=x_{j}^{2} \\
& x_{j}=\delta+j \Delta x=\delta+j h, \quad j=1,2,3, \cdots, N .
\end{aligned}
$$

Here $\Delta x=h$ denotes grid spacing in radial coordinate, $\delta$ is a small number $\left(10^{-6}\right.$ a.u., in present case), integer $j$ signifies the counter of increment in spatial direction, while $N$ is the 
total number of radial points. The operator $H$, given in spherical polar coordinates, by,

$$
H_{r}=-\frac{1}{2} \frac{d^{2}}{d r^{2}}-\frac{1}{r} \frac{d}{d r}+\left[\frac{\ell(\ell+1)}{2 r^{2}}+V(r)\right]=-\frac{1}{2} \frac{d^{2}}{d r^{2}}-\frac{1}{r} \frac{d}{d r}+v_{\mathrm{eff}}(r)
$$

can be recast in transformed $x$-grid as below (terms in the parenthesis correspond to $v_{\text {eff }}(r)$ ),

$$
H=-\frac{1}{8 x^{2}} D_{x}^{2}-\frac{3}{8 x^{3}} D_{x}+v_{\mathrm{eff}}(r)=a D_{x}^{2}+b D_{x}+v_{\mathrm{eff}}(r)
$$

In the above equation, $a=-\frac{1}{8 x^{2}}, b=-\frac{3}{8 x^{3}}, \ell$ signifies the angular momentum quantum number, while $D_{x}=\frac{d}{d x}, D_{x}^{2}=\frac{d^{2}}{d x^{2}}$ denote 1st, 2nd partial spatial derivatives. Such a radial grid has been found to be quite effective and successful for Coulombic systems [29, 30], for it provides a finer grid at small $r$ and coarser grid at large $r$. Subscripts in derivatives emphasize that these quantities are given in transformed grid $x$. In such a grid, Eq. (8) can be expressed as ( $j, n$ identify the increments in space and time coordinates respectively),

$$
\psi_{j}^{\prime(n+1)}=e^{-\Delta t H_{j}} \psi_{j}^{n},
$$

where a prime signifies an unnormalized diffusion function. This equation can be further written in an equivalent symmetric form, given below,

$$
e^{(\Delta t / 2) H_{j}} \psi_{j}^{\prime(n+1)}=e^{-(\Delta t / 2) H_{j}} \psi_{j}^{n} .
$$

By making use of Eq. (11), above equation can be further recast into a form, as below,

$$
e^{(\Delta t / 2)\left(a D_{x}^{2}+b D_{x}+v_{\mathrm{eff}}\right)} \psi_{j}^{\prime(n+1)}=e^{-(\Delta t / 2)\left(a D_{x}^{2}+b D_{x}+v_{\mathrm{eff}}\right)} \psi_{j}^{n} .
$$

Finally, expanding the exponentials, truncating them after second terms, followed by an approximation of $D_{x}$ and $D_{x}^{2}$ by two- and three-point difference formulas as below,

$$
\begin{aligned}
& D_{x} \psi_{j}^{n} \approx \frac{\psi_{j+1}^{n}-\psi_{j-1}^{n}}{\Delta x} \\
& D_{x}^{2} \psi_{j}^{n} \approx \frac{\psi_{j-1}^{n}-2 \psi_{j}^{n}+\psi_{j+1}^{n}}{2(\Delta x)^{2}},
\end{aligned}
$$

a set of $N$ simultaneous equations are obtained as follows:

$$
\alpha_{j} \psi_{j-1}^{\prime(n+1)}+\beta_{j} \psi_{j}^{\prime(n+1)}+\gamma_{j} \psi_{j+1}^{\prime(n+1)}=\xi_{j}^{n} .
$$

where the quantities $\alpha_{j}, \beta_{j}, \gamma_{j}, \zeta_{j}^{n}$ are identified as,

$$
\begin{aligned}
& \alpha_{j}=-\frac{\Delta t}{16 x_{j}^{2} h^{2}}+\frac{3 \Delta t}{32 x_{j}^{3} h}, \quad \beta_{j}=1+\frac{\Delta t}{8 x_{j}^{2} h^{2}}+\frac{\Delta t}{2} v_{\text {eff }}, \quad \gamma_{j}=-\frac{\Delta t}{16 x_{j}^{2} h^{2}}-\frac{3 \Delta t}{32 x_{j}^{3} h}, \\
& \zeta_{j}^{n}=\left(\frac{\Delta t}{16 x_{j}^{2} h^{2}}-\frac{3 \Delta t}{32 x_{j}^{3} h}\right) \psi_{j-1}^{n}+\left(1-\frac{\Delta t}{8 x_{j}^{2} h^{2}}-\frac{\Delta t}{2} v_{\text {eff }}\right) \psi_{j}^{n}+\left(\frac{\Delta t}{16 x_{j}^{2} h^{2}}+\frac{3 \Delta t}{32 x_{j}^{3} h}\right) \psi_{j+1}^{n} .
\end{aligned}
$$


Note that since discretization and truncation occurs on both sides of Eq. (14), cancellation of error may occur. Here, $\psi_{j-1}^{\prime(n+1)}, \psi_{j}^{\prime(n+1)}, \psi_{j+1}^{\prime(n+1}$ denote the unnormalized diffusion functions at time $t_{n+1}$ at radial grids $x_{j-1}, x_{j}, x_{j+1}$ respectively. The quantities $\alpha_{j}, \beta_{j}, \gamma_{j}$ and $\xi_{j}^{n}$ are identical to those appearing in [30] except the obvious differences in $v_{\text {eff }}$. For the sake of completeness, however, we provide them here. As can be seen, these are expressed in terms of $x_{j}^{2}, x_{j}^{3}$, as well as the space and time spacings $\Delta x, \Delta t$, while $v_{\text {eff }}$ entering in $\beta_{j}$ and $\xi_{j}^{n}$ only. Also note that $\xi_{j}^{n}$ requires knowledge of $\psi_{j-1}^{n}, \psi_{j}^{n}, \psi_{j+1}^{n}$, the normalized diffusion functions at radial grids $x_{j-1}, x_{j}$ and $x_{j+1}$ respectively at time step $t_{n}$. Equation (16) may further be rewritten in a convenient, tridiagonal matrix form,

$$
\left[\begin{array}{cccccc}
\beta_{1} & \gamma_{1} & & & & (0) \\
\alpha_{2} & \beta_{2} & \gamma_{2} & & & \\
& \ddots & \ddots & \ddots & & \\
& & \ddots & \ddots & \ddots & \gamma_{N-1} \\
(0) & & & & \alpha_{N} & \beta_{N}
\end{array}\right]\left[\begin{array}{c}
\psi_{1}^{\prime(n+1)} \\
\psi_{2}^{\prime(n+1)} \\
\vdots \\
\psi_{N-1}^{\prime(n+1)} \\
\psi_{N}^{\prime(n+1)}
\end{array}\right]=\left[\begin{array}{c}
\xi_{1}^{n} \\
\xi_{2}^{n} \\
\vdots \\
\xi_{N-1}^{n} \\
\xi_{N}^{n}
\end{array}\right] .
$$

This can be efficiently solved for $\left\{\psi_{j}^{\prime(n+1)}\right\}$ by using a modified Thomas algorithm [46].

Overall procedure of the calculation then involves following sequence of steps. At time step $n=0$, an initial guess of the wave function $\psi_{j}^{0}$ is made for all $j$. This is then propagated in accordance with Eq. (8) following the procedure described above to obtain $\psi^{\prime}(n+1)$ at $(n+1)$ th time step. At each time step, the wave function becomes smaller as $r$ assumes large values and finally tends to zero as $r$ goes to infinity. Setting them to zero for large $r$ was also found to be equally good provided it covered a sufficiently long radial distance. For an excited state calculation, $\psi_{j}^{0}$ needs to be orthogonalized to all lower states. Several orthogonalization schemes are available; here we have employed the widely used Gram-Schmidt method [47]. It is known that, while for smaller number of states the method is generally accurate, with increase in number of states, however, this tends to introduce numerical inaccuracy. Since in present work we are mostly concerned with ground and low-lying states, this therefore causes no significant impact on the results obtained. Then $\psi^{\prime(n+1)}$ is normalized to $\psi^{(n+1)}$ and the energy expectation values calculated as $\epsilon_{0}=\left\langle\psi^{(n+1)}|H| \psi^{(n+1)}\right\rangle$. If the difference in energy between two consecutive time steps, $\Delta \epsilon=\langle H\rangle^{(n+1)}-\langle H\rangle^{n}$, drops below a certain prescribed limit, then the diffusion function $\psi_{j}^{(n+1)}$ is stored as the corresponding solution of Hamiltonian $H$. Otherwise, $\psi_{j}^{(n+2)}$ is calculated and above steps repeated until $\Delta \epsilon$ reaches 
TABLE I: Calculated eigenvalues, radial expectation values, normalization and virial ratios for six lowest states of spherical quantum harmonic oscillator corresponding to $\ell=0$.

\begin{tabular}{lllllll}
\hline \hline Energy $^{a}$ & $\left\langle r^{-2}\right\rangle$ & $\left\langle r^{-1}\right\rangle$ & $\left\langle r^{0}\right\rangle$ & $\left\langle r^{1}\right\rangle$ & $\left\langle r^{2}\right\rangle$ & $\langle V\rangle /\langle T\rangle$ \\
\hline 1.49999999 & 2.000000 & 1.128379 & 1.000000 & 1.128379 & 1.499999 & 0.999999 \\
3.4999999 & 2.000000 & 0.940316 & 1.000000 & 1.692569 & 3.499999 & 0.999999 \\
5.4999999 & 1.99999 & 0.83688 & 1.000000 & 2.11571 & 5.50000 & 1.000000 \\
7.4999999 & 2.00000 & 0.76770 & 0.99999 & 2.46833 & 7.49999 & 0.999999 \\
9.499999 & 1.99998 & 0.71668 & 1.00000 & 2.77689 & 9.49999 & 1.00001 \\
11.499999 & 2.0000 & 0.67678 & 1.00000 & 3.05456 & 11.5000 & 1.00000 \\
\hline \hline
\end{tabular}

a The exact energies [49] of six states are $1.5,3.5,5.5,7.5,9.5$ and 11.5 respectively.

such limit. In this study, a tolerance of $10^{-12}$ was set for energy while 10001 radial grid points were used uniformly. Once the diffusion function reaches the desired convergence in this way, $\psi^{\prime(n+1)}$ is normalized to $\psi^{(n+1)}$, from which the various properties of interest, such as the expectation values, etc., may be obtained as: $\langle A\rangle^{(n+1)}=\left\langle\psi^{(n+1)}|A| \psi^{(n+1)}\right\rangle$. Note that, for excited-state calculation, diffusion function must remain orthogonal to all lower states at all time steps, not just at initial time step. Continuing this procedure, one could then obtain first excited state $\epsilon_{1}$, second excited state $\epsilon_{2}$, third excited state $\epsilon_{2}$, and so on. The grid spacing was adjusted according to the problem, as dictated by nature of the particular potential under study. This is mentioned at appropriate places in the discussion that follow. Overlap and energy integrals at each time step were evaluated by standard Newton-Cotes quadrature, while finite-difference formulas were used for the spatial derivatives [48].

\section{RESULTS AND DISCUSSION}

At first, we present some specimen results to test the validity and performance of our method. First one is the familiar 3D spherical quantum harmonic oscillator, which is an exactly solvable system. Table I gives 6 lowest states corresponding to rotational quantum number $\ell=0$. At this point it is to be noted that all results reported in all tables throughout the article are truncated and not rounded-off. Therefore, all the entries are taken to be correct up to the place they are presented. These calculations are performed in a radial box size of 10 a.u. Not very exhaustive, but a few sample calculations were made to gauge the variations with respect to grid parameters. In general, good-quality results could be 
obtained with even smaller number of points, such as 501 or so, and accuracy could be improved even further (from reported values) by 2-3 decimal places by increasing number of radial points from current values. These are briefly touched upon in a following paragraph. However, we have not made any attempt to optimize the grid here, as our primary objective in this work is to demonstrate the capability and appropriateness of this method in context of physically important situations. As already known, energy levels of isotropic harmonic oscillator are given by: $E_{k, \ell}=\left(k+\ell+\frac{3}{2}\right)=\left(m+\frac{3}{2}\right)$ a.u., where $k$ is zero or any even positive integer, $\ell$ can be zero or any positive integer, so that $m$ can take on all integral values, zero or positive. Therefore the two quantum numbers $\ell, m$ must have same parity [49]. We see that the present results are in excellent agreement with exact values for all states. Additionally, the position expectation values of these states in columns 2-6 can also be obtained analytically. We have verified $\left\langle r^{-2}\right\rangle,\left\langle r^{-1}\right\rangle,\left\langle r^{1}\right\rangle$ and $\left\langle r^{2}\right\rangle$ for the first two states. For ground state $(k=m=0 ; \ell=0)$ these are: $2, \frac{2}{\sqrt{\pi}}, \frac{2}{\sqrt{\pi}}$ and $\frac{3}{2}$, while for first excited state $(k=m=2 ; \ell=0)$, these values are $2, \frac{5}{3 \sqrt{\pi}}, \frac{3}{\sqrt{\pi}}, \frac{15}{2}$ respectively. Present calculated values are in good agreement with these estimates. As a further test on quality of our eigenfunctions, numerically obtained normalization and virial ratios are also provided in fourth and last columns respectively. For the $n$th stationary state of a 3D quantum harmonic oscillator, the latter can be obtained from,

$$
\frac{d}{d t}\langle\mathbf{r} \cdot \mathbf{p}\rangle=\frac{i}{\hbar}\langle[H, \mathbf{r} \cdot \mathbf{p}]\rangle=2\langle T\rangle-\langle\mathbf{r} \cdot \nabla V\rangle=2\langle T\rangle-2\langle V\rangle=0
$$

so that $\frac{\langle V\rangle}{\langle T\rangle}=1$. This further establishes the reliability and strength of our present method.

In Table II, we examine two more special cases where exact analytical results are available. First one is the so-called Morse potential having following functional form [51]:

$$
V(r)=25\left(e^{-4(r-3)}-2 e^{-2(r-3)}\right), \quad E_{n}=-\left[5-\sqrt{2}\left(n+\frac{1}{2}\right)\right]^{2}, \quad n=0,1,2,3 .
$$

Morse potential plays a very significant role in the vibration-rotation spectra of diatomic molecules and has been extensively studied by a large number of workers ever since its inception about 85 years ago. The above potential supports only four bound states; corresponding exact analytical energies are given in Eq. (20) [51]. In the left panel, our energies for all four states are seen to match exactly with these as well as B-spline result [52] and generalized pseudospectral method [21]. It is worth mentioning that for first three states the precision of Table II could be reached quite easily with $r_{\max }=20$ a.u. only, while same 
TABLE II: Calculated eigenvalues (a.u.) in Morse potential (left panel) and ground states of charged harmonic oscillator (right panel) along with literature results. PR signifies Present Result.

\begin{tabular}{|c|c|c|c|c|c|}
\hline \multicolumn{3}{|c|}{ Morse oscillator } & \multicolumn{3}{|c|}{ Charged harmonic oscillator $(\alpha=1)$} \\
\hline$n$ & Energy (PR) & Energy (Reference) & $\lambda$ & Energy (PR) & Energy $\left(\right.$ Exact $\left.^{a}\right)$ \\
\hline$\overline{0}$ & -18.42893218 & $-18.42893218^{b, c, d}$ & 0 & 1.49999999 & 1.5 \\
\hline 1 & -8.2867965 & $-8.2867965^{b, c, d}$ & 2 & 2.499999999 & 2.5 \\
\hline 2 & -2.1446609 & $-2.1446609^{b, c, d}$ & $\sqrt{20}$ & 3.499999999 & 3.5 \\
\hline \multirow[t]{4}{*}{3} & -0.002525 & $-0.002525^{b, c, d}$ & $\sqrt{30+6 \sqrt{17}}$ & 4.499999999 & 4.5 \\
\hline & & & $\sqrt{70+6 \sqrt{57}}$ & 5.499999999 & 5.5 \\
\hline & & & 14.450001026966 & 6.500000000 & 6.5 \\
\hline & & & 18.503131410003 & 7.500000000 & 7.5 \\
\hline
\end{tabular}

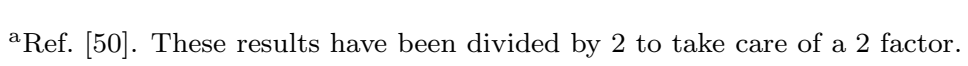

${ }^{b}$ Exact result, Ref. [51]. $\quad{ }^{c}$ B-Splines result, Ref. [52]. $\quad{ }^{\mathrm{d}}$ Generalized pseudospectral result, Ref. [21].

for the fourth state requires a value of about 200 a.u. Our second example corresponds to a special case of a general class of interaction potentials, known as spiked harmonic oscillators (SHO), characterized by the following functional form,

$$
V(r)=\frac{1}{2}\left[r^{2}+\frac{\lambda}{r^{\alpha}}\right], \quad \alpha>0 .
$$

In this equation, coupling parameter $\lambda$ determines strength of perturbative potential, while positive constant $\alpha$ defines type of singularity at origin. In a relatively simpler case of $\alpha=1$ (termed as charged harmonic oscillator), the system does not exhibit super-singularity and the Hamiltonian assumes a simplified confined Coulomb potential type form effectively. It has been pointed out that such a system offers an infinite set of elementary solutions. The right panel compares seven such elementary solutions in ground state of a charged harmonic oscillator along with exact results [53]. Note, the first one $(\lambda=0)$ refers to trivial case of an unperturbed Hamiltonian, i.e., a quantum harmonic oscillator having energy $E=3 / 2$. The other $\lambda$ 's are taken from solutions of the polynomial equation [53]. All these ground states are obtained by engaging a radial grid of 10 a.u. In all these instances, current energies match excellently with exact values.

Once the accuracy and reliability is established, next in Table III, we report first 6 states of a charged harmonic oscillator belonging to angular quantum number $\ell=0$. All these states are obtainable from an $r_{\max }=20$ a.u. A broad range of the coupling parameter, viz., $\lambda= \pm 0.001, \pm 0.01, \pm 0.1, \pm 1, \pm 10$ is considered, covering a wide interaction region. 
TABLE III: Calculated eigenvalues and selected expectation values (a.u.) of charged harmonic oscillator for several positive and negative values of $\lambda$. First six eigenstates corresponding to $\ell=0$ are given. Numbers in the parentheses are quoted from Ref. [20].

\begin{tabular}{|c|c|c|c|c|c|c|c|}
\hline$\lambda$ & Energy & $\left\langle r^{-1}\right\rangle$ & $\left\langle r^{1}\right\rangle$ & $\lambda$ & Energy & $\left\langle r^{-1}\right\rangle$ & $\left\langle r^{1}\right\rangle$ \\
\hline \multirow[t]{6}{*}{-0.001} & $1.4994357(1.4994357)$ & 1.12854 & 1.12826 & \multirow[t]{6}{*}{0.001} & $1.5005641(1.5005641)$ & 1.12822 & 1.12850 \\
\hline & $3.4995298(3.4995298)$ & 0.94038 & 1.69251 & & $3.5004701(3.5004701)$ & 0.94025 & 1.69262 \\
\hline & $5.4995815(5.4995815)$ & 0.83692 & 2.11567 & & $5.5004184(5.5004184)$ & 0.83685 & 2.11575 \\
\hline & 7.4996161 & 0.76772 & 2.46830 & & 7.5003838 & 0.76768 & 2.46836 \\
\hline & 9.4996416 & 0.71670 & 2.77685 & & 9.5003583 & 0.71667 & 2.77689 \\
\hline & 11.4996616 & 0.67679 & 3.05454 & & 11.5003383 & 0.67676 & 3.05458 \\
\hline \multirow[t]{6}{*}{-0.01} & 1.4943542 & 1.12994 & 1.12720 & \multirow[t]{6}{*}{0.01} & 1.5056380 & 1.12682 & 1.12955 \\
\hline & 3.4952968 & 0.94093 & 1.69201 & & 3.5047000 & 0.93970 & 1.69313 \\
\hline & 5.4958147 & 0.83723 & 2.11535 & & 5.5041835 & 0.83654 & 2.11607 \\
\hline & 7.4961609 & 0.76793 & 2.46806 & & 7.5038379 & 0.76748 & 2.46860 \\
\hline & 9.4964161 & 0.71684 & 2.77666 & & 9.5035830 & 0.71652 & 2.77708 \\
\hline & 11.4966158 & 0.67690 & 3.05438 & & 11.5033835 & 0.67665 & 3.05473 \\
\hline \multirow[t]{6}{*}{-0.1} & $1.4431875(1.4431875)$ & 1.14420 & 1.11659 & \multirow[t]{6}{*}{0.1} & $1.5560334(1.5560334)$ & 1.11304 & 1.14008 \\
\hline & $3.4528298(3.4528298)$ & 0.94649 & 1.68698 & & $3.5468614(3.5468614)$ & 0.93414 & 1.69818 \\
\hline & $5.4580701(5.4580701)$ & 0.84031 & 2.11209 & & $5.5417576(5.5417576)$ & 0.83342 & 2.11935 \\
\hline & 7.4615591 & 0.76993 & 2.46565 & & 7.5383286 & 0.76544 & 2.47102 \\
\hline & 9.4641260 & 0.71827 & 2.77475 & & 9.5357939 & 0.71507 & 2.77900 \\
\hline & 11.4661312 & 0.67797 & 3.05280 & & 11.5338082 & 0.67555 & 3.05632 \\
\hline \multirow[t]{6}{*}{-1} & 0.8926027 & 1.31029 & 1.00677 & \multirow[t]{6}{*}{1} & 2.0289385 & 0.99421 & 1.24072 \\
\hline & 3.0145292 & 1.00105 & 1.63746 & & 3.9548368 & 0.87947 & 1.74928 \\
\hline & 5.0733048 & 0.86907 & 2.08009 & & 5.9096008 & 0.80118 & 2.15283 \\
\hline & 7.1108547 & 0.78819 & 2.44200 & & 7.8779872 & 0.74381 & 2.49574 \\
\hline & 9.1379189 & 0.73109 & 2.75599 & & 9.8541170 & 0.69938 & 2.79855 \\
\hline & 11.1588046 & 0.68756 & 3.03725 & & 11.8351717 & 0.66356 & 3.07246 \\
\hline \multirow[t]{6}{*}{-10} & $-12.440500(-12.440499)$ & 5.02341 & 0.29792 & \multirow[t]{6}{*}{10} & $5.2887417(5.2887417)$ & $0.57934^{a}$ & $1.88860^{b}$ \\
\hline & $-2.4172388(-2.4172388)$ & 1.46634 & 1.04083 & & $7.0754394(7.0754394)$ & $0.57219^{a}$ & $2.20351^{b}$ \\
\hline & $0.8696992(0.8696992)$ & 0.98099 & 1.69717 & & $8.8981164(8.8981164)$ & $0.56237^{a}$ & $2.49563^{b}$ \\
\hline & 3.4282462 & 0.82558 & 2.16774 & & 10.7479670 & 0.55143 & 2.76737 \\
\hline & 5.7738977 & 0.74308 & 2.54292 & & 12.6187932 & 0.54019 & 3.02143 \\
\hline & 8.0207630 & 0.68873 & 2.86318 & & 14.5061493 & 0.52906 & 3.26020 \\
\hline
\end{tabular}

a Literature values of $\left\langle r^{-1}\right\rangle$ for first three states are: $0.579336,0.572186,0.562375$ 20].

b Literature values of $\left\langle r^{1}\right\rangle$ for first three states are: 1.888604, 2.203514, 2.495625 [20].

For $\lambda= \pm 0.001, \pm 0.1$ and \pm 10 , the first three states have been calculated before through a generalized pseudospectral method [20]. Current energies obtained from imaginary-time evolution technique are in quite good agreement with these literature values, quoted here in parentheses. While the current results do not reach the precision of [20] within our present 
TABLE IV: First six lowest eigenvalues (a.u.) of charged harmonic oscillator corresponding to $l=0$, for two values of $\lambda$, with variations in grid. $N_{r}$ implies number of radial points.

\begin{tabular}{cccccc}
\hline \hline$\lambda$ & $n$ & $N_{r}=501$ & $N_{r}=1001$ & $N_{r}=2001$ & $N_{r}=5001$ \\
\hline-0.001 & 0 & 1.4994081 & 1.4994275 & 1.4994337 & 1.4994357 \\
& 1 & 3.4994911 & 3.4995136 & 3.4995271 & 3.4995298 \\
& 2 & 5.4995407 & 5.4995717 & 5.4995793 & 5.4995815 \\
& 3 & 7.4995801 & 7.4996020 & 7.4996125 & 7.4996161 \\
& 4 & 9.4996278 & 9.4996374 & 9.4996400 & 9.4996416 \\
0.01 & 11.4996001 & 11.4996553 & 11.4996598 & 11.4996616 \\
& 5 & 5.2887423 & 5.2887418 & 5.2887417 & 5.2887417 \\
& 0 & 7.0754429 & 7.0754398 & 7.0754394 & 7.0754394 \\
& 1 & 8.8981276 & 8.8981178 & 8.8981166 & 8.8981164 \\
& 2 & 10.7479984 & 10.7479717 & 10.7479674 & 10.7479670 \\
& 3 & 12.6188822 & 12.6188020 & 12.6187939 & 12.6187932 \\
& 4 & 14.5063045 & 14.5061641 & 14.5061505 & 14.5061493 \\
\hline \hline
\end{tabular}

implementation, these are certainly still very good and almost for all practical purposes, sufficiently accurate. No other results are available at this time for other states. In addition, for each of these states, the position expectation values $\left\langle r^{-1}\right\rangle$ and $\langle r\rangle$ are given as well.

At this point, Table IV gives a comparison of energies obtained in various grids. For this purpose, two $\lambda$ values of -0.001 and 10 of the charged harmonic oscillator are selected. All six eigenvalues are considered for four $N_{r}$ values, viz., 501, 1001, 2001 5001, keeping the initial guess same in all occasions. It is clearly seen that, even the smallest grid produces results accurate up to fourth place of decimal except the highest state corresponding to $\lambda=10$. For all the states, however, the results improve with successive increase in $N_{r}$, i.e., a denser grid is needed. The simulation box was roughly 15 a.u. As already mentioned, while such tests are not undertaken for all the potentials under study, it has, however, been verified that, the last grid $N_{r}=5001$ is sufficient to completely reproduce all the eigenvalues of previous table for these two $\lambda$ values. Nevertheless, from the experience of these two cases, we believe this could be equally true for the other potential sets in the table as well.

As a last example, Table $\mathrm{V}$ reports ground-state energies of SHO for two values of $\alpha=4$ (left) and 6 (right) for small as well as large $\lambda_{\mathrm{s}}$. Note that in the last three decades, there has been significant interest in this system due to its many fascinating characteristics. One distinctive feature of such a potential is that once the perturbation $\lambda|r|^{-\alpha}$ is turned on, it is impossible to completely turn off the interaction. Also, in the region of $\alpha \geq 5 / 2$, it 
TABLE V: Calculated ground-state energies E (in a.u.) of the SHO with $\alpha=4$ and 6 for several values of $\lambda$. The literature results are divided by a 2 factor. PR signifies Present Result.

\begin{tabular}{|c|c|c|c|c|}
\hline \multirow[t]{2}{*}{$\lambda$} & \multicolumn{2}{|r|}{ Energy $(\alpha=4)$} & \multicolumn{2}{|r|}{ Energy $(\alpha=6)$} \\
\hline & $\mathrm{PR}$ & Literature & PR & Literature \\
\hline 0.001 & 1.53438158 & $1.53438158^{a, b}, 1.534385^{c}$ & 1.63992791 & $1.63992791^{a, b}$ \\
\hline \multirow[t]{2}{*}{0.005} & 1.57417615 & $1.57417615^{b, d}$ & 1.71144209 & $1.71144209^{b}, 1.71144208^{d}$ \\
\hline & & $1.574175^{e}, 1.574195^{f}$ & & $1.71144^{e}, 1.71151^{f}$ \\
\hline \multirow[t]{2}{*}{0.01} & 1.60253374 & $1.60253374^{a, b}, 1.60254^{c}$ & 1.75272613 & $1.75272613^{a, b}, 1.752726195^{d}$ \\
\hline & & $1.60253374^{d}, 1.602535^{e, g}, 1.602635^{f}$ & & $1.752725^{e}, 1.75287^{f}, 1.7527265^{g}$ \\
\hline 0.05 & 1.71258069 & $1.71258069^{b}$ & 1.88277010 & $1.88277010^{b}$ \\
\hline 0.1 & 1.78777599 & $1.78777599^{a, b}, 1.787785^{c}, 1.787775^{g}$ & 1.95783261 & $1.95783261^{b}$ \\
\hline 0.5 & 2.06529243 & $2.06529243^{b}$ & 2.19395453 & $2.19395453^{b}$ \\
\hline 1 & 2.24708899 & $2.24708899^{a, b}, 2.24709^{c, g}$ & 2.32996998 & $2.32996998^{a, b}, 2.329970^{g}$ \\
\hline 5 & 2.89222177 & $2.89222177^{b}, 2.89222^{g}$ & 2.75657950 & $2.75657950^{b}, 2.7565795^{g}$ \\
\hline 10 & 3.30331125 & $3.30331125^{a, b, h}, 3.30331^{c, g}$ & 3.00160451 & $3.00160451^{a, b}, 3.0016045^{g}, 3.00160451^{h}$ \\
\hline 50 & 4.73277787 & $4.73277787^{b}$ & 3.76776072 & $3.76776072^{b}$ \\
\hline 100 & 5.63254021 & $5.63254021^{a, b}, 5.63254^{c}, 5.6325402^{h}$ & 4.20667914 & $4.20667914^{b, h}$ \\
\hline 500 & 8.73793385 & $8.73793385^{b}$ & 5.57607711 & $5.57607711^{b}$ \\
\hline 1000 & 10.6847312 & $10.6847312^{a, b, h}, 10.68473^{c}$ & 6.35930853 & $6.35930853^{b}$ \\
\hline
\end{tabular}

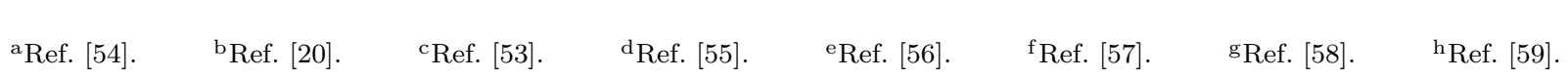

exhibits super-singularity. For many other facets of this potential, the reader is referred to the following references [20, 53 60], Both the $\alpha$ values considered can lead to supersingularity; these have been studied by numerous analytic, semi-analytic as well as numerical methodologies. Some of these literature results are given here for comparison. It is seen that the present methodology offers results which are in good agreement with these. The most accurate results are those from analytic continuation method [54] and generalized pseudospectral method [20]. The present energies are not superior to these, but still are excellent and evidently better than many other reference values.

Finally, to show the quality of our wave functions obtained, we depict the radial distribution functions of charged harmonic oscillator in Fig. 1. Diagrams (a)-(e) in left panel correspond to the potential (a) (with $\alpha=1, \lambda=0.01$ ) and first four low-lying state densities respectively, with (b) referring to that of ground state. Similarly in (f)-(j) in right panel, plots for potential (f) (with $\alpha-1, \lambda=-10$ ) and four lowest states are displayed, with (g) identifying the lowest state. In both cases, density plots for all states are given in same scale of radial distance. They both carry the signatures of acceptable eigenfunctions with number 
of nodes increasing with state index. It is seen that, in the right side, peak height decreases to a greater extent as one goes to higher excitations, compared to the potential in left side.

A few words may be devoted to the initial trial function. Most of our calculations were performed with Gaussian-type functions as starting guess to launch the computations. However, several other sample guessed (including some wild) functions were tried to test the efficiency of this formalism. In such cases, the effective computation time required to achieve convergence of desired accuracy varies with initial guess, as more and more iterations are required. Generally, it was found that, keeping all things unchanged, during iterative process, mixing diffusion function with that from previous time step by a certain percentage (we employed a 50:50 mixture) increased the rate of convergence. Accuracy of the present method depends on density of the grid and propagation time. The degeneracy in case of symmetric and non-symmetric 2D double-well oscillators [35] as well as pseudo-degeneracy in 1D double wells [31, 33] have been well represented by this method. It is conceivable that convergence and accuracy of our results could be further improved by choosing different spatial grid, more appropriate and suitable initial wave functions, higher-order finite difference schemes as well as higher precision computation, some of which may be taken up later.

\section{CONCLUSION}

Energy eigenvalues, select position expectation values and probability densities of 3D spherically symmetric potentials are obtained accurately and efficiently by means of an imaginary time evolution method in conjunction with minimization of an energy expectation value. Numerical propagation of the resulting diffusion equation eventually hits ground state and ensuring orthogonalization to lower states, leads to excited states in a sequential manner. Comparison with available literature data reveals that good-quality, meaningful results could be produced in all the occasions concerned. Thus it could pose a viable alternative to the existing methodologies available for such systems. This is illustrated for a variety of systems, such as quantum harmonic oscillator, Morse potential, charged harmonic oscillator and spiked harmonic oscillator. The present work, as such, remains valid for spherically symmetric potentials. And therefore may not be directly applicable to situations, where the same is not possible, such as that in [61]. Another disconcerting feature of the method lies in the fact that for excited state calculations it must maintain orthogonality 

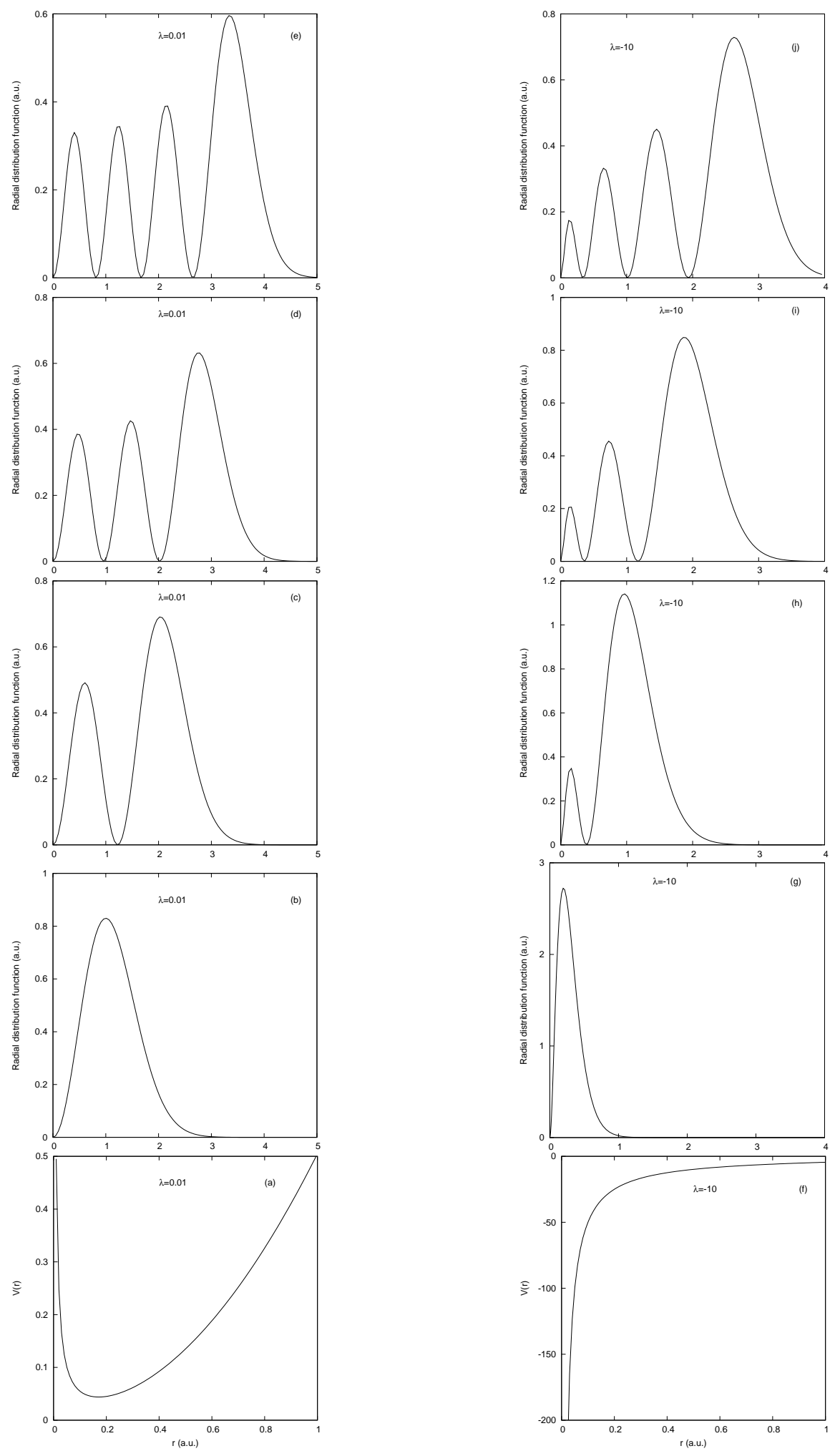

FIG. 1: The radial probability distribution functions of charged harmonic oscillators. Left and right panels corresponds to $\lambda=0.01$ and -10 . The two potentials are shown in $\{(a),(f)\}$, while $\{(b),(g)\} ;\{(c),(h)\} ;\{(d),(i)\} ;\{(e),(j)\}$ refer to the densities of ground, first, second and third excited states respectively, corresponding to $\ell=0$. 
requirement with respect to all other lower states of same symmetry. That means, unless all the lower-state wave functions are properly converged in the active grid space, accurate results would be difficult for excited states. Future applications of the method to non-zero angular states, as well as other interacting potentials of physical interest, such as molecular, atomic, screening, power-law, rational etc., and quantum confinement studies may further consolidate the success of this approach.

\section{ACKNOWLEDGMENT}

It is gratefully acknowledged. It is a pleasure to thank Mr. Siladitya Jana for supplying some of the references.

[1] K. J. Oyewumi, Found. Phys. Lett. 18, 75 (2005).

[2] R. Sever, M. Bucurgat, C. Tezcan and Ö. Yeşiltaş, J. Math. Chem. 43, 749 (2008).

[3] S.-H. Dong and Z.-Q. Ma, Int. J. Mod. Phys. E 11, 155 (2002).

[4] R. Sever, C. Tezcan, M. Aktaş and Ö. Yeşiltaş, J. Math. Chem. 43, 845 (2008).

[5] L.-Y. Wang, X.-Y. Gu, Z.-Q.Ma and S.-H. Dong, Found. Phys. Lett. 15, 569 (2002).

[6] S.-H. Dong, R. Lemus and A. Frank, Int. J. Quant. Chem. 86, 433 (2002).

[7] S.-H. Dong and R. Lemus, Int. J. Quant. Chem. 86, 265 (2002).

[8] S.-H. Dong and J. García-Ravelo, Phys. Scr. 75, 307 (2007).

[9] W.-C. Qiang and S.-H. Dong, Phys. Lett. A 368, 13 (2007).

[10] H. Akcay and R. Sever, J. Math. Chem. 50, 1973 (2012).

[11] A. F. Nikiforov and V. B. Uvarov, Special Functions of Mathematical Physics, Berlin, Birkhauser, (1988).

[12] J. M. Fellows and R. A. Smith, J. Phys. A 42, 333503 (2009).

[13] H. Çiftçi, R. L. Hall and N. Saad, J. Phys. A 36, 11807 (2003).

[14] H. Çiftçi, R. L. Hall and N. Saad, Phys. Lett. A 340, 388 (2005).

[15] Z. Q. Ma and B. W. Xu, Europhys. Lett. 69, 685 (2005).

[16] Z. Q. Ma and B. W. Xu, Int. J. Mod. Phys. E 14, 599 (2005).

[17] S. H. Dong, Factorization Method in Quantum Mechanics, Springer, (2007). 
[18] S.-H. Dong, Int. J. Theor. Phys. 40, 559 (2001).

[19] S.-H. Dong, Found. Phys. Lett. 15, 385 (2002).

[20] A. K. Roy, Phys. Lett. A 321, 231 (2004).

[21] A. K. Roy, J. Phys. G 30, 269 (2004).

[22] W.-C. Qiang and S.-H. Dong, Europhys. Lett. 89, 10003 (2010).

[23] F. A. Serrano, X.-Y. Gu an S.-H. Dong, J. Math. Phys. textbf51, 082103 (2010).

[24] J. B. Anderson, J. Chem. Phys. 63, 1499 (1975).

[25] J. B. Anderson, J. Chem. Phys. 65, 4121 (1976).

[26] D. R. Garmer and J. B. Anderson, J. Chem. Phys. 86, 4025 (1987).

[27] R. Kosloff and H. Tal-Ezer, Chem. Phys. Lett. 127, 223 (1986).

[28] M. D. Feit, J. A. Fleck Jr. and A. Steiger, J. Comput. Phys. 47, 412 (1982).

[29] B. K. Dey and B. M. Deb, J. Chem. Phys. 110, 6229 (1999).

[30] A. K. Roy, B. K. Dey and B. M. Deb, Chem. Phys. Lett. 308, 523 (1999).

[31] A. K. Roy and S. I. Chu, J. Phys. B 35, 2075 (2002).

[32] A. K. Roy, N. Gupta and B. M. Deb, Phys. Rev. A 65, 012109 (2002).

[33] N. Gupta, A. K. Roy and B. M. Deb, Pramana-J. Phys. 59, 575 (2002).

[34] A. Wadehra, A. K. Roy and B. M. Deb, Int. J. Quant. Chem. 91, 597 (2003).

[35] A. K. Roy, A. J. Thakkar and B. M. Deb, J. Phys. A 38, 2189 (2005).

[36] I. W. Sudiarta and D. J. Wallace Geldart, J. Phys. A 40, 1885 (2007).

[37] I. W. Sudiarta and D. J. Wallace Geldart, Phys. Lett. A 372, 3145 (2008).

[38] I. W. Sudiarta and D. J. Wallace Geldart, J. Phys. A 42, 285002 (2009).

[39] M. Strickland and D.Yager-Elorriaga, J. Comput. Phys. 229, 6015 (2010).

[40] L. Lehtovaara, J. Toivanen and J. Eloranta, J. Comput. Phys. 221, 148 (2007).

[41] P. J. J. Luukko and E. Räsänen, Comput. Phys. Comm. 184, 769 (2013).

[42] S. A. Chin, Phys. Lett. A 226, 344 (1997).

[43] S. A. Chin, S. Janecek and E. Krotscheck, Chem. Phys. Lett. 470, 342 (2009).

[44] M. Aichinger, S. A. Chin and E. Krotscheck, Comput. Phys. Comm. 171, 197 (2005).

[45] B. L. Hammond, W. A. Lester Jr. and P. J. Reynolds, Monte Carlo Methods in Ab Initio Quantum Chemistry, World Scientific, Singapore, (1994).

[46] W. H. Press, S. A. Teukolsky, W. T. Vetterling and B. P. Flannery, Numerical Recipes: The Art of Scientific Computing, 3rd Edition, Cambridge Univ. Press, New York (2007). 
[47] W. H. Greub, Linear Algebra, 4th Edition, Springer (1981).

[48] M. Abramowitz and I. Stegun (Ed.) Handbook of Mathematical Functions, Dover, New York (1964).

[49] C. Cohen-Tannoudji, B. Diu and F. Laloë, Quantum Mechanics, Wiley-VCH, (1992).

[50] V. C. Aguilera-Navarro, F. M. Fernández, R. Guardiola and J. Ros, J. Math. Phys. 25, 6379 (1992).

[51] B. W. Shore, J. Chem. Phys. 58, 3855 (1973).

[52] M. Landtman, Phys. Lett. A 175, 147 (1993).

[53] V. C. Aguilera-Navarro, A. C. Coelho and N. Ullah, Phys. Rev. A 491477 (1994).

[54] E. Buendiá, F. J. Gálvez and A. Puertas, J. Phys. A 286731 (1995).

[55] W. Solano-Torres, G. A. Estévez, F. M. Fernández and G. C. Groenenboom, J. Phys. A 25 3427 (1992).

[56] J. Killingbeck, J. Phys. B 15829 (1982).

[57] L. C. Detwiler and J. R. Klauder, Phys. Rev. D 111436 (1975).

[58] F. M. Fernández, Phys. Lett. A 160511 (1991).

[59] C. R. Handy, Phys. Lett. A 21615 (1996).

[60] F. J. Gómez and J. Sesma, J. Phys. A 43, 385302 (2010).

[61] F. A. Fertig and W. Kohn, Phys. Rev. A 62, 052511 (2000). 